"Buscar la verdad y afianzar los valores transcendentales", misión de las universidades en su artículo primero, inspirado en los principios humanísticos. Ley de Universidades 8 de septiembre de 1970.
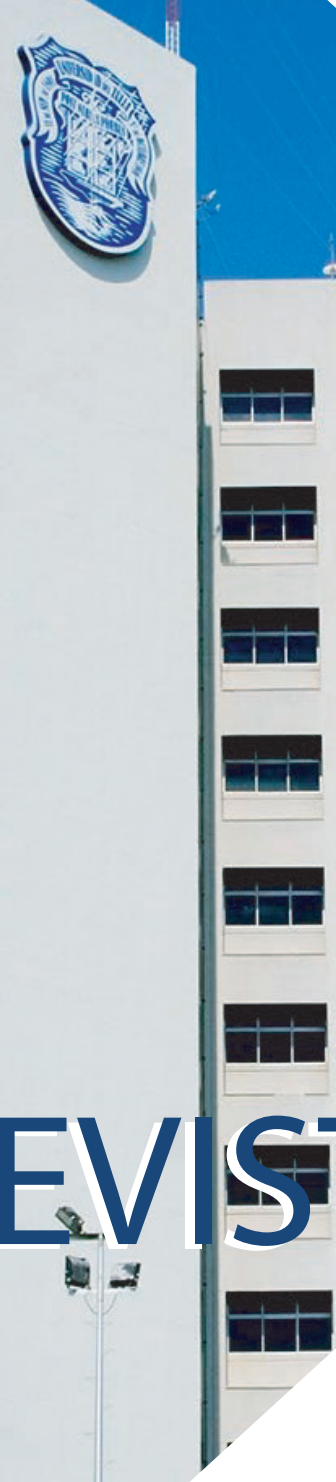


\title{
Influencia de las partículas de caucho en la resistencia a la compresión de bloques de concreto
}

\author{
Edison Javier Lara Guerrero $\mathbb{D}$, David Patricio Guerrero Cuasapaz $\mathbb{D}$, Byron Iván \\ Altamirano León $\mathbb{D}$
}

Universidad Politécnica Salesiana, Facultad de Ingeniería Civil, Av. Rumichaca Ñan y Av. Moran Valverde, Casilla 17-12-536, Quito-Ecuador

*Autor de correspondencia: dguerrero@ups.edu.ec

https://doi.org/10.22209/rt.v43n3a03

Recepción: 18/09/2019 | Aceptación: 29/06/2020 | Publicación: 01/09/2020

\section{Resumen}

La problemática en la disposición de residuos de caucho crea la necesidad de proponer nuevas alternativas, el enfoque es la mitigación del impacto ambiental generado por la contaminación de los neumáticos, aprovechando este material reciclado se propone la utilización en materiales para la construcción. La investigación consiste en el diseño y elaboración de un bloque de concreto prototipo utilizando partículas de caucho, para diferentes porcentajes de sustitución por agregado fino, que posea similares características técnicas y económicas de un bloque de concreto convencional tipo B, propuesto en la norma NTE INEN 3066 2016-11. Lo propuesto, es la sustitución de porcentajes (10\%, 15\% y 20\%) en volumen de agregado fino por partículas de caucho producto de la trituración de neumático. Los resultados exponen que la alternativa es viable bajo los parámetros antes mencionados; por lo tanto, el bloque de concreto con partículas de caucho del 20\% de sustitución mostró una resistencia neta mínima a la compresión simple 3,69 MPa cumpliendo con lo establecido. El precio del bloque de concreto prototipo con partículas de caucho del $20 \%$ de sustitución resulta más económico que un bloque convencional tipo B propuesto en esta investigación.

Palabras Clave: bloque prototipo; caucho reciclado; concreto; resistencia a la compresión.

\section{Influence of rubber particles on the compressive strength of concrete blocks}

\begin{abstract}
The problem in the disposal of rubber particles, creates a necessity to propose new alternatives to approach the mitigation of the environmental impact generated by the contamination of tires. In this sense, taking advantage of this recycled material is proposed for use in construction materials. This investigation consists in the design and elaboration of a prototype concrete block using rubber particles, for different percentages of substitutions by fine aggregate that has similar technical and economic characteristics of a conventional type B concrete block, proposed in the standard NTE INEN $30662016-11$. The proposal is the replacement of percentages (10\%,15\% and $20 \%)$ in volume of fine aggregates by rubber particles product of the crushing of tire. The results show that the alternative is viable under the parameters mentioned above; therefore, the concrete block with $20 \%$ rubber particles showed a minimum net compression strength of $3.69 \mathrm{MPa}$ complying with the established. The price of the prototype concrete block with $20 \%$ rubber particles is cheaper than a conventional block type B proposed in this research.
\end{abstract}

Keywords: prototype block; recycled rubber; concrete; compression resistance. 


\section{Introducción}

El incremento de la población genera mayor solicitación de viviendas tanto en el sector rural y urbano generando demanda de materiales para la construcción.

Las diferentes técnicas de construcción nacen por la gran necesidad de aprovechar los elementos que se encuentran en nuestro alrededor, mejor cuando estos están causando un impacto ambiental como son los neumáticos de un vehículo; por tal razón en los últimos años se ha observado como los profesionales de la construcción incluyen ciertos elementos a los materiales construcción.

La cantidad de vehículos en circulación se incrementa año a año, en consecuencia, se genera subproductos derivados de los vehículos, tal es el caso de los desechos que provienen de procesos mecánicos del recauche de los neumáticos [1].

El problema ambiental de los desechos de llantas se genera por el escaso conocimiento de planes de gestión de residuos, tanto por temas culturales como por la falta de políticas gubernamentales que intervienen en empresas privadas, investigaciones sobre la reutilización y disposición final de este tipo de residuos.

En el Ecuador, ante la falta de la aplicación de una medida política, que indique qué hacer con los neumáticos que dejan de ser útiles para circular, el Ministerio del Ambiente del Ecuador (MAE) puso en marcha el Plan de Gestión Integral de los Neumáticos Usados, con el fin de reducir la contaminación ambiental que el producto causa, el Acuerdo Ministerial de 1998, que en su parte pertinente dispone que los comerciantes de llantas deben recuperar el $30 \%$ de su mercado.

El estado considera a los neumáticos como desechos especiales, debido a que su combustión produce nubes tóxicas y además pueden ser fuentes de propagación de epidemias trasmitidas por mosquitos [2].

Debido a esto se hace necesario generar ideas que permitan dar soluciones a este tipo de problema, como es el aprovechamiento de incluir partículas de caucho triturado de neumáticos vehiculares a los bloques de concreto de esta manera lograr minimizar el impacto ambiental e incrementar las ganancias al producir estos especímenes.

En la investigación realizada por Torres [3], "Se concluyó que la resistencia mecánica (compresión) se redujo con los tres porcentajes en volumen adicionado de residuos de caucho. Se valoran las propiedades mecánicas del concreto cambiando en un 10\%, 20\% y 30\% el volumen del agregado fino con residuos de llantas de caucho".

Por otra parte, en la investigación realizada por Bastidas P. y Viñán M. [4], "La resistencia a la compresión alcanzada a los 28 días de las mezclas de concreto referentes al tamaño de partículas de caucho que pasan por el tamiz $\mathrm{N}^{\circ} 16, \mathrm{~N}^{\circ} 30$ y $\mathrm{N}^{\circ} 50$ fueron $69,95 \%, 82,65 \%$ y $80,36 \%$ con respecto a la mezcla convencional de concreto que obtuvo el $100 \%$ de resistencia a compresión con la cual fue diseñada la mezcla; por lo tanto la mezcla que mejor comportamiento tuvo fue el concreto elaborado con partículas retenidas en el tamiz $\mathrm{N}^{\circ} 30^{\prime \prime}$, cabe mencionar que la cita de esta investigación es para aprovechar los resultados obtenidos de las partículas donde se obtuvo la mejor resistencia.

La investigación de Nazer A. et al [1] indica que "en los ensayos de compresión y flexo tracción, se constató que el concreto testigo presentó el mejor comportamiento a la compresión a los 28 días"; además expone: que es evidente la viabilidad de fabricar concretos que posean una adecuada resistencia a la compresión con la inclusión de varios tipos de fibras entre las cuales se tiene las partículas de neumáticos que se encuentran fuera de uso.

Con la finalidad de analizar la influencia de la adición del caucho granulado proveniente de los neumáticos en desuso, como parte del agregado fino en la fabricación de bloques huecos de concreto, a través de ensayos destructivos y no destructivos, indica que la adición de caucho hasta en un $20 \%$ no presentan variaciones significativas al compararlos con el concreto tradicional. Por otra parte, el módulo de rigidez dinámico disminuye a mayor adición de caucho granulado, además el caucho granulado en el concreto ofrece mayor aislamiento acústico y térmico [5].

Otro ejemplo claro es lo recuperado de una pista de aterrizaje producto de la fricción del neumático y superficie de rodamiento, como material modificador de una mezcla asfáltica que utiliza asfalto AC-20 [6].

Existe una buena compatibilidad entre las partículas de neumáticos fuera de uso y el concreto, "su mejora respecto de la fisuración por retracción y de disipación de energía elástica, lo que redundaría en una reducción del nivel sonoro del tráfico" [7], investigación utilizada en el diseño de firmes o bases de concreto utilizando fibras de caucho.

Este artículo de investigación se elaboró con la finalidad de analizar y evaluar la incidencia de sustituir las partículas de caucho provenientes de neumáticos reciclados por el agregado fino y determinar resultados que serán influyentes en el aspecto ambiental y económico.

\section{Metodología}

La investigación se realizó en la ciudad de Quito, provincia de Pichincha, Ecuador. Las partículas de caucho utilizadas para este trabajo fueron recolectadas en Durallanta al sur de la cuidad, el análisis de las propiedades físicas de las partículas se realizó en el Laboratorio de Ensayo de Materiales de la Universidad Politécnica Salesiana.

Se realizaron tres tipos de mezclas de concreto con tamaños de partículas de caucho retenidos en 
los tamices (ver Tabla 1) y una mezcla convencional (Resistencia neta mínima a la compresión en bloques de concreto tipo B promedio resistencia neta mínima $=4,0$ 3,5 MPa).

Tabla 1. Tamices utilizados en el cribado del caucho.

\begin{tabular}{cc}
\hline Tamiz ASTM (\#) & Tamaño de partículas $(\mathbf{m m})$ \\
\hline 4 & 4,760 \\
8 & 2,380 \\
10 & 2,000 \\
12 & 1,700 \\
16 & 1,190 \\
30 & 0,596 \\
\hline
\end{tabular}

\section{Caracterización del material}

\section{Cemento.}

El cemento que se utilizó es de la marca ARMADURO 囚, Tipo IP (uso general) ASTM C 595, con una densidad de $3.1 \mathrm{~g} / \mathrm{cm}^{3}$, en concordancia con la norma NTE INEN 490, con peso específico de $2,92 \mathrm{~g} / \mathrm{cm}^{3}$ [8]

Agua.

Para la mezcla se utilizó agua potable a temperatura ambiente $\left(13^{\circ} \mathrm{C}-23^{\circ} \mathrm{C}\right)$.

\section{Agregados.}

Se utilizaron materiales pétreos provenientes de la cantera de Pifo, Provincia de Pichincha norma NTE INEN 696 (ASTM C 136-06). [9]

\section{Granulometría.}

La granulometría de los agregados finos y gruesos se realizó bajo la norma NTE INEN 872 (ASTM C 33-08), cumpliendo todo lo especificado [10].

\section{Colorimetría en el agregado fino.}

Este ensayo se realizó para determinar el color, en base de las impurezas orgánicas que contiene el árido fino dando una clasificación del 1 al 5. NTE INEN 855 (ASTM C 94, ICONTEC 3318) [11].

\section{Peso unitario de los agregados.}

Este ensayo se realizó bajo los parámetros de la norma NTE INEN 858 (ASTM C 29-09) indicados en Tabla 2, para determinar los pesos unitarios del agregado fino y material grueso [12].
Tabla 2. Caracterización de los Agregados.

\begin{tabular}{ccc}
\hline Propiedad & $\begin{array}{c}\text { Agregado } \\
\text { fino }\end{array}$ & $\begin{array}{c}\text { Agregado } \\
\text { grueso }\end{array}$ \\
\hline Módulo de finura & 3,01 & $*$ \\
Tamaño máximo nominal (mm) & 4,76 & 19,05 \\
Porcentaje de Abrasión (\%) & $*$ & 32,6 \\
P. Unitario Compactado (kg/m3) & 1640 & 1440 \\
P. Específico Sólidos (kg/m3) & 2660 & 2650 \\
Contenido de Humedad (\%) & 7,24 & 1,44 \\
Porcentaje de Absorción (\%) & 6,30 & 2,67 \\
\hline
\end{tabular}

Densidad relativa y absorción del árido fino.

Este ensayo se fundamenta en la norma NTE INEN 856, 857 (ASTM C 128-07a, ASTM C127-07) donde describe la determinación de la densidad relativa y absorción del agregado fino [13].

\section{Proceso de reciclado de caucho de neumáticos}

Pasos para el proceso de reciclado Durallanta:

\section{Inspección inicial}

En este primer paso el técnico realiza una inspección más rigurosa, determinando daños severos en el neumático que influyen en la aprobación o rechazo del mismo.

\section{Raspado}

En este paso, se retira el piso remanente del neumático (ver Figura 1), con equipos de precisión se prepara la llanta para recibir la banda apropiada según las especificaciones dadas por Durallanta (Radio de raspado).

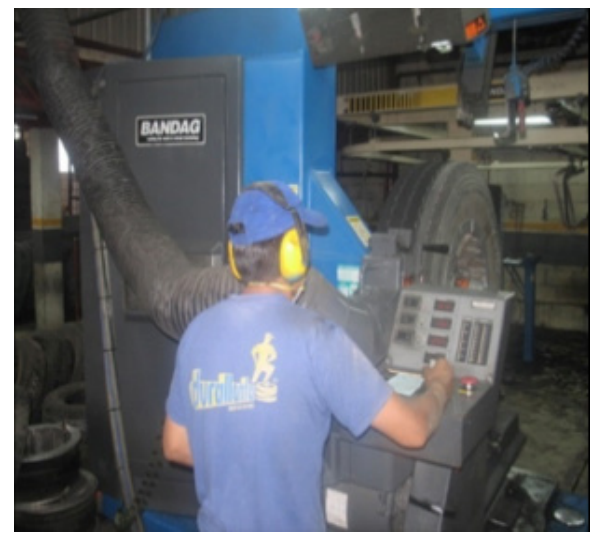

Figura 1. Máquina de aspas para el proceso de raspado. 


\section{Recolección del proceso de raspado}

En este paso todas las partículas provenientes del raspado de los neumáticos se recolectan mediante conductos impulsados por una bomba de aire hacia la zona de recolección (ver Figura 2), donde se encuentra la materia prima que se utiliza para la elaboración de los bloques prototipo.

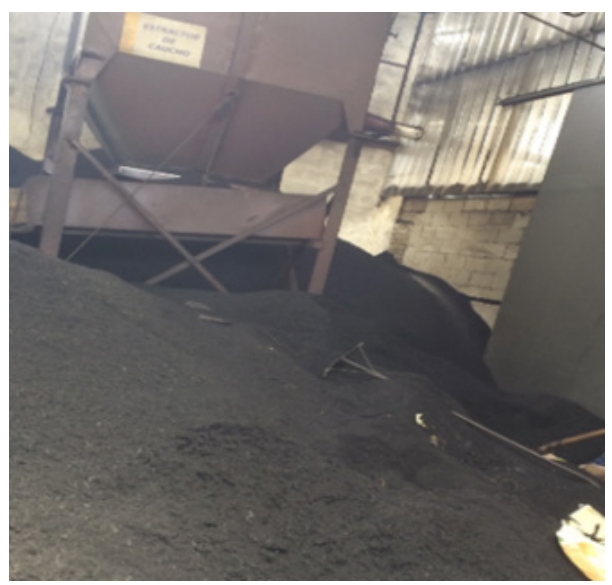

Figura 2. Extractor de caucho.

\section{Diseño de concreto}

La mezcla para el concreto convencional se realizó de acuerdo con el A.C.I.211 Esta norma utiliza las propiedades de los agregados y la resistencia a la compresión requerida [14].

Elaboración de bloques de concreto prototipo utilizando partículas de caucho

Para la elaboración de los bloques prototipos de concreto se utilizaron moldes de $390 \mathrm{~mm}$ x $190 \mathrm{~mm} \times 140$ $\mathrm{mm}$ requerido por la norma NTE INEN 3066 (ASTM C 90).

El muestreo de los bloques prototipos de concreto se realizó bajo la norma NTE INEN 2859-1 (ISO 2859-1); para determinar el asentamiento de la mezcla se utilizó el cono de Abrams NTE INEN 1778, cuyo valor sirve para medir la trabajabilidad de la mezcla [15].

El procedimiento de curado en la fábrica, es mantener los bloques durante los primeros siete días en las condiciones de humedad, mojándolos en la mañana y en la tarde, cubriéndolos del sol con una manta húmeda de algodón.

Una forma de curar los bloques es rociarlos con agua, utilizando mangueras (preferiblemente con atomizador) de manera que no se sequen en ningún momento. Otra forma de curarlos es recubrirlos con brines o mantas de algodón que sean mojadas permanentemente, o con láminas de plástico que formen un ambiente hermético que evite la pérdida de humedad por evaporación. La cobertura con plásticos negros y exposición al sol, acelera el desarrollo de resistencia siempre que los bloques se mantengan húmedos [16].

La obtención de las partículas de caucho para los bloques prototipo, se realizó con los tamices que se exponen en la Tabla 1, al realizar la mezcla se tomó el material pasante del tamiz $\mathrm{N}^{\circ} 10$ y retenido en el tamiz $\mathrm{N}^{\circ} 12, \mathrm{~N}^{\circ} 16, \mathrm{~N}^{\circ} 30$ (ver Figura 3), se realizó esto para aprovechar el máximo del material reciclado.

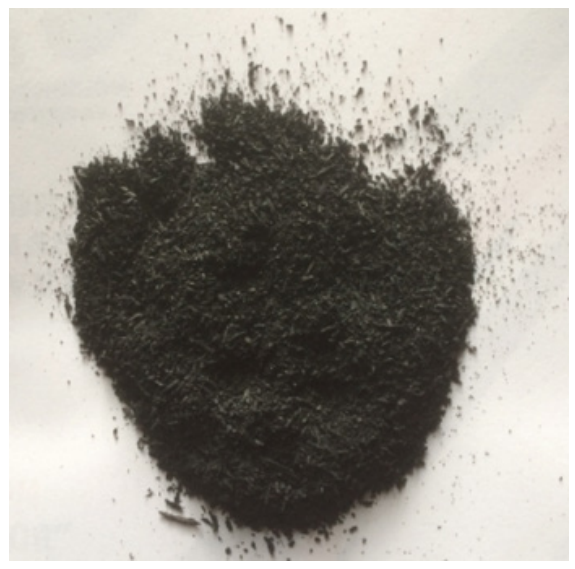

Figura 3. Tamaño de partículas de caucho retenido tamiz $\mathrm{N}^{\circ} 12, \mathrm{~N}^{\circ} 16, \mathrm{~N}^{\circ} 30$ (ver Tabla 1 ).

Con estas partículas de caucho reciclado después de realizar el análisis granulométrico (ver Figura 4), se fabricaron los prototipos, sustituyendo el volumen del agregado fino por partículas de caucho retenidos por los tamices anteriormente descritos.

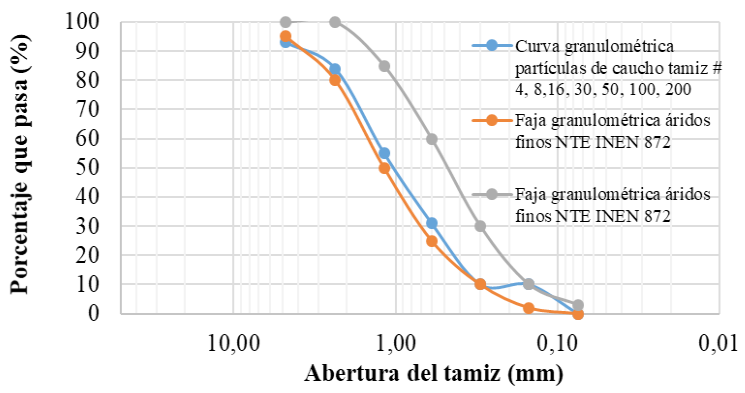

Figura 4. Curva granulométrica del caucho reciclado.

Ensayo de compresión realizado a la edad 28 días, para este propósito se ensayaron tres bloques convencionales tipo B no estructural y 40 bloques prototipo. 
Los bloques de concreto convencionales tipo B no estructurales y los bloques de concreto prototipo para el ensayo a la compresión simple han sido unidades enteras donde su área neta se puede determinar mediante el procedimiento descrito en el numeral D.5.5 de la norma NTE INEN 3066 para el ensayo a la compresión simple. Se debe considerar el área neta de los especímenes enteros, registrando la carga máxima alcanzada por cada probeta, para luego obtener el esfuerzo promedio de la mezcla convencional y mezclas con colocación de caucho; este procedimiento se encuentra normado en el anexo E de la norma NTE INEN 3066. El nivelado de los especímenes fue realizado de acuerdo a la norma NTE INEN 2619 (ASTM C 1552-09a) [15].

\section{Resultados y Discusión}

\section{Resultados de caracterización}

Los áridos del Sector de Pifo seleccionados para esta investigación no se encuentran dentro de la faja NTE INEN 872. [10], para lo cual se tuvo que utilizar el método de FULLER THOPSON [14], para el ajuste de la curva granulométrica y estimar el contenido de arena y grava dando como resultado el $51 \%$ de grava y $49 \%$ de arena, es un agregado con un mayor porcentaje de partículas gruesas, esto afecta la demanda de agua y la trabajabilidad del concreto, sin embargo, la variación referente a la norma no es significativa para descartar el uso de este material.

En el análisis de impurezas orgánicas en la norma NTE INEN 855 en el agregado fino se visualizó un color amarillento claro, puede usarse en concretos de alta resistencia [11].

La dosificación o proporción del material tuvo una relación de 1:7:7 (cemento - árido grueso - árido fino) en volumen. Se variaron los porcentajes de sustitución de las partículas de caucho reciclado de la siguiente manera; $10 \%, 15 \%$ y $20 \%$ en volumen por áridos fino.

Para el cálculo volumétrico de diseño de la mezcla se tomó como referencia el volumen de un saco de cemento de $50 \mathrm{~kg}$ mediante el uso de un recipiente de acero diseñado de $0,045 \mathrm{~m}^{3}$ para el efecto, en la que fue necesario graduarla en su parte interior y poder calcular los porcentajes de las partículas de caucho reciclados para realizar la preparación de la mezcla, previa la elaboración de los bloques de concreto.

La Tabla 3 muestra las cantidades de los materiales necesarios para la mezcla de concreto. Para esta dosificación, se necesitan 5,6 recipientes de volumen de árido fino por cada saco de cemento, ya que se sustituyó el $20 \%$ obteniendo un valor de 1,4 recipientes de partículas de caucho para la elaboración de los bloques prototipos.
Tabla 3. Dosificación en volumen utilizada para la elaboración del bloque prototipo utilizando el $20 \%$ de partículas de caucho en sustitución por áridos finos.

\begin{tabular}{ccccc}
\hline Material & $\mathbf{m}^{3}$ & $\mathbf{L}$ & Recipiente & Cemento \\
\hline Cemento & & & & 1 Saco \\
Arena & 0,252 & 5,6 & \\
Grava & 0,315 & 7 & \\
Agua & 0,048 & 48,00 & & \\
$\begin{array}{c}\text { Recipiente de } \\
\text { caucho 20\% }\end{array}$ & 0,063 & & 1,4 & \\
$\begin{array}{c}\text { Nota: Recipiente de } \\
0,045 \mathrm{~m}^{3}\end{array}$ & & & & \\
\hline
\end{tabular}

El mezclado se realizó de manera mecánica a través de una mezcladora industrial de $0,70 \mathrm{~m}^{3}$ de capacidad adquirida por la fábrica donde se elaboraron los bloques prototipo.

Una vez mezclados los materiales, se procede a colocar en la máquina vibradora o bloquera. La duración del vibrado, así como la potencia del motor de la máquina vibradora son factores que influyen notablemente en la resistencia de los bloques (aprox. 20 segundos de vibración por molde).

Cuando se coloca la mezcla de concreto en la bloquera, ésta se compacta y se consolida a base de presión y vibración controladas (ver Figura 5). El método de llenado se debe realizar en capas y con la ayuda de una pala se puede ir acomodando la mezcla.

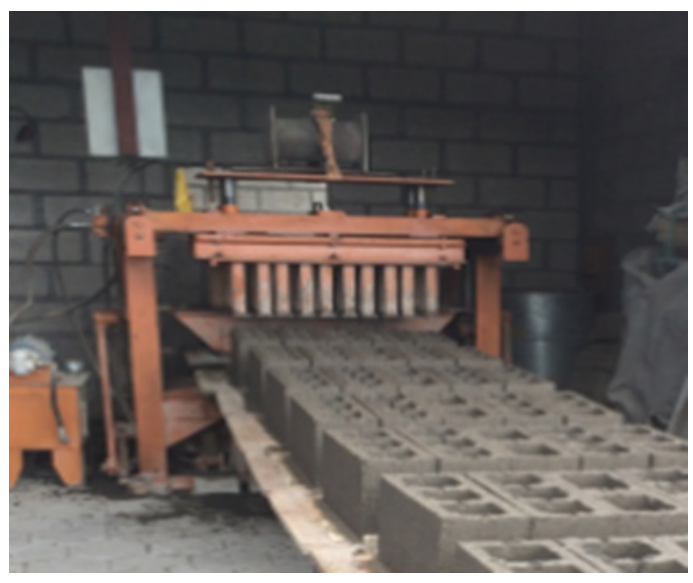

Figura 5. Máquina vibradora o bloquera.

\section{Resultados de los ensayos a compresión}

El análisis del comportamiento a la compresión 
de los bloques de concreto convencional y prototipos con partículas de caucho $10 \%, 15 \%$ y $20 \%$, se ensayaron a los 28 días de su elaboración.

En la Tabla 4 se muestra el esfuerzo promedio a la compresión de las cuatro muestras elaboradas.

Tabla 4. Resistencia a la Compresión de Bloques.

\begin{tabular}{cccccc}
\hline Muestra & $\begin{array}{c}\text { Edad } \\
\text { (días) }\end{array}$ & $\begin{array}{c}\text { Bloque } \\
\text { Convencional } \\
\text { tipo b (MPa) }\end{array}$ & $\begin{array}{c}\text { Bloque } \\
\text { prototipo } \\
\mathbf{1 0 \%} \\
\text { (MPa) }\end{array}$ & $\begin{array}{c}\text { Bloque } \\
\text { prototipo } \\
\mathbf{1 5 \%} \\
\text { (MPa) }\end{array}$ & $\begin{array}{c}\text { Bloque } \\
\text { prototipo } \\
\mathbf{2 0 \%} \\
\text { (MPa) }\end{array}$ \\
\hline 1 & 28 & 6,24 & 5,24 & 4,89 & 3,92 \\
2 & 28 & 5,52 & 5,19 & 5,22 & 3,41 \\
3 & 28 & 5,77 & 5,08 & 5,09 & 3,79 \\
Promedio & 28 & 5,84 & 5,17 & 5,06 & 3,71 \\
\hline
\end{tabular}

El bloque convencional sin adición de polvo de caucho diseñado y elaborado en la fábrica alcanzó una resistencia promedio de 5,84 MPa se observó que la resistencia promedio de los bloques prototipos con partículas de caucho del $10 \%, 15 \%$ y $20 \%$ es de 5,17 5,06 y 3,71 MPa respectivamente (ver Figura 6), entonces se eligió el bloque prototipo que tiene el $20 \%$ de partículas de caucho (mayor aprovechamiento de partículas) y cumple con la resistencia establecida para un bloque tipo B no estructural para mampostería que se está planteando en esta investigación.

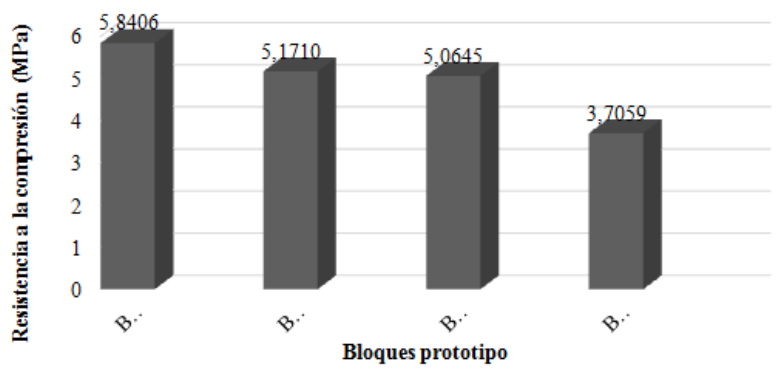

Figura 6. Resistencia a la compresión simple.

La sustitución del agregado fino con las partículas de caucho que tiene densidad y resistencia menor al árido fino hace que las características mecánicas del concreto disminuyan (ver Figura 7), por tanto, la resistencia de un material compuesto como el concreto, depende de la resistencia de sus componentes [17].

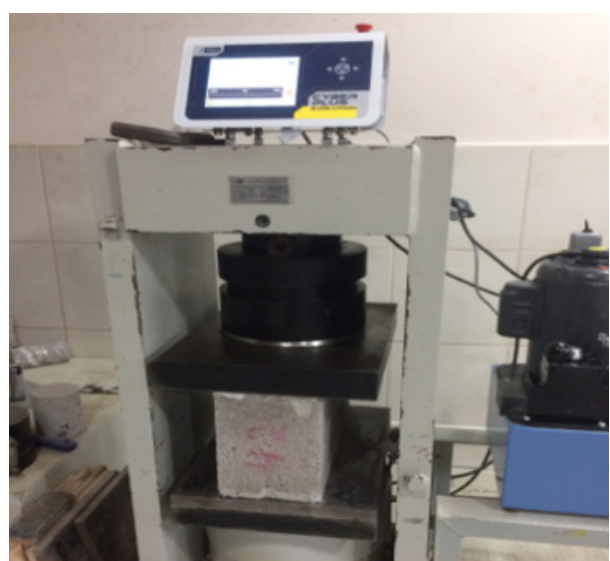

Figura 7. Bloque prototipo ensayo de compresión.

La disminución de resistencia a la compresión a los 28 días de edad de los diferentes tipos de bloques ensayados con los porcentajes del 10\%, 15\% y $20 \%$ de partículas de caucho fueron 11,46\%, 13,29\% y 36,55\%, respectivamente (ver Figura 8), con respecto al 100\% de la resistencia alcanzada por el bloque convencional.

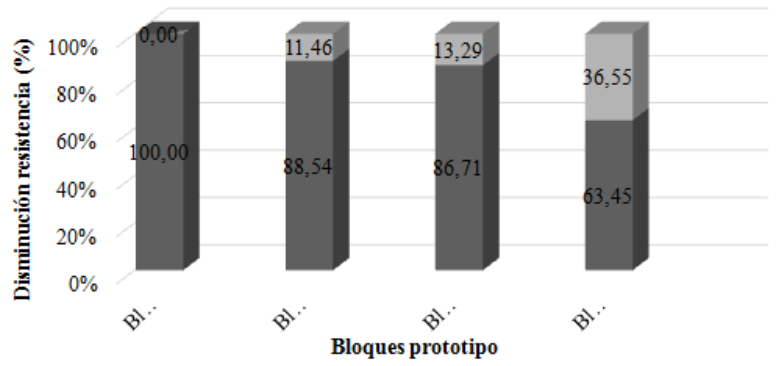

Figura 8. Disminución de la resistencia respecto al bloque convencional tipo B no estructural.

\section{Selección del Prototipo}

Una vez identificado el porcentaje óptimo que cumple con la resistencia a la compresión simple se obtuvo una población de 70 especímenes, para ello se tomó una muestra con más del $50 \%$ según L. P. J. Puerto [18], cuando no hay investigaciones realizadas anteriormente al tipo de tema que se está realizando se debe colocar $50 \%$ de proporción deseada y $50 \%$ de proporción no deseada. Entonces se verificaron 40 bloques prototipos en dimensiones, aspectos visuales y resistencia a la compresión simple, a fin de determinar los datos probabilísticos del prototipo.

Este tipo de población se considera finita. La población finita según R. Tulio [19], los elementos en su totalidad son identificables por el investigador, desde 
el punto de vista del conocimiento que se tiene sobre la cantidad total, es decir cuando el investigador cuenta con el registro de todos los elementos que conforman la investigación en estudio.

$\mathrm{Al}$ analizar estos datos obtenemos los siguientes resultados para la resistencia media un valor de 3,7 MPa y una desviación estándar de 0,35 esta es una medida del grado de dispersión de los datos analizados con respecto al valor promedio entonces interpretamos que el grado de dispersión es menor a 1; como se indica en la Figura 9, entonces estos datos son confiables para la investigación propuesta según R. Tulio [19].

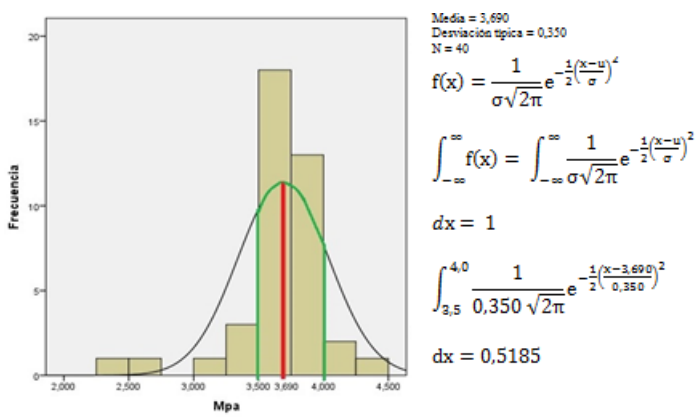

Figura 9. Campana de Gauss distribución normal.

Al determinar la campana de Gauss el resultado de los 40 especímenes es del 51,85\% del total de las muestras ensayadas que se encuentran dentro de la resistencia buscada $(3,5-4,0) \mathrm{MPa}$.

\section{Costos de fabricación de los bloques analizados}

Los costos obtenidos para la fabricación del bloque convencional tipo B no estructural y un bloque de concreto prototipo sustituyendo partículas de caucho al $20 \%$, con la dosificación establecida 1:7:7, y 1:7:5,6 respectivamente se resume en lo siguiente:

En la Figura 10 se presenta el costo de elaboración del bloque convencional tipo B tiene un valor de \$ 0,39 USD (dólares estadounidense) y el costo del bloque prototipo es de \$ 0,38 USD.

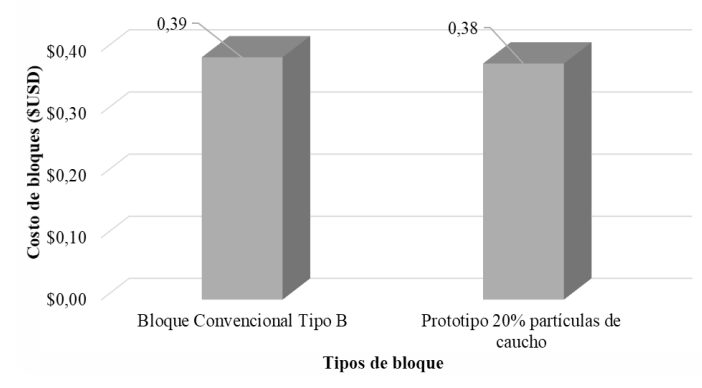

Figura 10. Costo de bloques.
Como podemos evidenciar el costo del bloque convencional tipo B es superior al prototipo, esto se debe a que se reemplazó el material fino por partículas de caucho obteniendo así el ahorro de un centavo de dólar. Para la presente investigación se obtuvo un lote de 70 especímenes dando como resultado un ahorro indicado anteriormente, por lo tanto se evidencia que en la producción diaria se estima la utilización de 80 sacos de cemento en consecuencia se obtendrá 5600 blocks, es decir, la producción anual de blocks será de 2044000 especímenes, dando como resultado un ahorro en la producción anual correspondiente a \$20 440 dólares americanos. El ahorro económico anual es muy significativo, pues incentiva incrementar la producción para lograr mayor utilidad en la Empresa Ecuatoriana, además de contribuir indirectamente con la descontaminación ambiental bajando el índice de impacto ambiental que provoca el caucho en su proceso de descomposición.

\section{Conclusiones}

El uso de las partículas del caucho reciclado retenido en los tamices $\mathrm{N}^{\circ} 12, \mathrm{~N}^{\circ} 16$ y $\mathrm{N}^{\circ} 30$ fue para optimizar y aprovechar el máximo material reciclado de la fábrica de Durallanta y contribuir a disminuir el impacto ambiental que actualmente se registra en la ciudad.

El bloque de concreto convencional tipo B sometido al ensayo de compresión simple tuvo un desempeño de $(5,84 \mathrm{MPa})$ para la cual fue diseñada, el bloque prototipo con sustitución de partículas de caucho del $10 \%, 15 \%$ y $20 \%$ fue de 5,17 5,06 y 3,71 $\mathrm{MPa}$ respectivamente, por tanto, el bloque de concreto prototipo utilizando el $20 \%$ de sustitución partículas de caucho fue el escogido por que cumple con la resistencia a la compresión neta mínima de la norma NTE INEN 3066 requerida en esta investigación.

La disminución de resistencia a la compresión a los 28 días de edad del bloque de concreto prototipo ensayado con $20 \%$ de partículas de caucho fue de $36,55 \%(3,71 \mathrm{MPa})$, con respecto al $100 \%(5,84 \mathrm{MPa})$ de la resistencia alcanzada por el bloque de concreto convencional tipo B, a pesar de que se dió esta reducción, el bloque prototipo está dentro de la resistencia requerida.

Se obtuvo una desviación estándar de 0,35 y coeficientes de variación menores a 1 para todas las variables, lo que significa que los resultados fueron homogéneos y con un grado de dispersión bajo. Por lo que se determina que el porcentaje óptimo de partículas de caucho, es del $20 \%$ respecto al volumen de árido fino utilizado en la mezcla, y se logró la resistencia a la compresión deseada para la investigación.

Dentro del análisis de precios unitarios para elaboración de los bloques de concreto convencionales y prototipo, se tiene que el bloque prototipo es más 
económico, esto se debe a que se reemplazó un porcentaje del árido fino por las partículas de caucho por árido fino obteniendo un valor inferior en ese rubro. El resto de los materiales, mano de obra y equipo son similares.

El precio unitario de bloque de concreto convencional tipo B para la dosificación planteada tiene un mayor valor en el mercado, mientras que el prototipo tiene un menor valor; la factibilidad, la relación entre costos y beneficios son adecuados por lo expuesto anteriormente, el análisis de precios unitarios se concluyó finalmente que hay un ahorro económico del bloque de concreto prototipo con $20 \%$ de partículas de caucho con respecto al bloque convencional tipo $\mathrm{B}$.

Finalmente, en esta investigación se demostró que el bloque prototipo cumple la norma NTE INEN 3066, también ayuda a mitigar el impacto ambiental producido por la contaminación de los neumáticos vehiculares desechados.

\section{Agradecimientos}

Los autores agradecen a la Universidad Politécnica Salesiana (Quito-Ecuador) en particular al Laboratorio de Ensayo de Materiales por el apoyo y gestión realizado en beneficio del avance académico de nuestra Carrera de Ingeniería Civil.

\section{Referencias Bibliográficas}

[1] Nazer A., Honores A., Chulak P.y Pavez O.: "Hormigón sustentable basado en fibras de neumáticos fuera de uso". Rev. Int. Contam. Ambie., Vol. 35, No. 3, (2019), $1-2$.

[2] Ministerio del Ambiente del Ecuador: "Instructivo para la Gestión Integral de Neumáticos Usados Acuerdo Ministerial 098”. Ecuador, (2015).

[3] Torres H.: "Valoración de las propiedades mecánicas y de durabilidad de concreto adicionado con residuos de llantas de caucho". Escuela Colombiana de Ingeniería Julio Garavito, (2014), 16-27.

[4] Bastidas P. y Viñán M.: "Análisis de las propiedades físicas y mecánicas del hormigón elaborado con partículas de caucho de neumáticos reciclados". Universidad Politécnica Salesiana. Quito, (2017), 1-9.

[5] Suárez I. y Mujica E.: "Bloques de concreto con material reciclable de caucho para obras de edificación". Universidad Nacional de San Antonio Abad del Cusco. Perú, (2016), 26-112.

[6] Cando W., Bonilla P., Yánez G., Bucheli J., Muñoz A., Orquera M., Fernández L. y Espinoza P.: "Efecto de la incorporación por vía seca de residuos de caucho obtenido tras su remoción de una pista de aterrizaje de aeropuerto en un asfalto AC-20". Rev. Téc. Ing.
Univ. Zulia. Vol. 43, No. 1, (2020), 3-11.

[7] Witoszek, B., Hernández, F., Alonso, M., Bollati, M., Parga, B., Barluenga, G., y Benito C.: "Hormigón con fibras de caucho de recuperación de neumáticos usados yde polipropileno diseño del firme de hormigón de caucho".Recuperado de:https://www. aecarretera.com/congresos/CL26.pdf, (2004), 1415.

[8] NTE INEN 409: "Cementos hidráulicos compuestos, Requisitos". Quito, (2011).

[9] NTE INEN 696: "Áridos, análisis granulométrico en los áridos, fino y grueso". Quito, (2011).

[10] NTE INEN 872: “Áridos para hormigón”. Quito, (2011).

[11] NTE INEN 855: “Áridos.Determinación de impurezas orgánicas en el árido fino para hormigón". Quito,(2010).

[12] NTE INEN 858: “Áridos. Determinación de la masa unitaria (peso volumétrico) y el porcentaje de vacíos". Quito, (2010).

[13] NTE INEN 856: "Áridos. Determinación de la densidad, densidad relativa (gravedad específica) y absorción del árido fino". Quito, (2010).

[14] Niño J.: "Tecnología del concreto: Materiales, Propiedades y Diseño de mezcla". Tomo 1, Tercera Edición, Impreso por Nomos Impresores. Bogotá, (2010), 184-205.

[15] NTE INEN 3066: “Bloques de hormigón. Requisitos y métodos de ensayo". Quito, (2016).

[16] Régil 0.: "Optimización del proceso de fabricación de bloques de concreto del estándar $15 \times 20 \times 40 \mathrm{~cm} 3$ con grado de resistencia $28 \mathrm{~kg} / \mathrm{cm}^{2}$, caso específico fuerte-block máquinas \#1 Y \#2". Universidad de San Carlos de Guatemala, (2015), 1-12.

[17] Cruz M. y GómezO.: "Influencia del agregado grueso reciclado de mampostería en el comportamiento del concreto". Tesis de Maestría, Pontificia Universidad Javeriana. Bogotá. http://hdl.handle. net/10554/12676, (2017), 28-102.

[18] Lagares P. y Puerto J.: "Población y Muestra Técnicas de muestreo". MaMaEuSch** Management Mathematics for European Schools 94342 - CP - 1 - 2001 - 1 - DE - COMENIUS - C21. Universidad de Sevilla, (2001), 2-13.

[19] Ramirez T.: "Como hacer un proyecto de investigación, de Técnicas de análisis y aspectos administrativos". Caracas, Editorial Panapo, (1999), 165-167. 


\section{REVISTA TECNICA}

DE LA FACULTAD DE INGENIERIA

UNIVERSIDAD DEL ZULIA

Vol. 43. $\mathrm{N}^{\circ} 3$, Septiembre - Diciembre 2020 pp. 114 - 176

Esta revista fue editada en formato digital y publicada el 31 de Agosto de 2020, por el Fondo Editorial Serbiluz, Universidad del Zulia. Maracaibo-Venezuela

www.luz.edu.ve

www.serbi.luz.edu.ve

www.produccioncientifica.org 